\title{
Ретинопатия недоношенных. Обзор
}

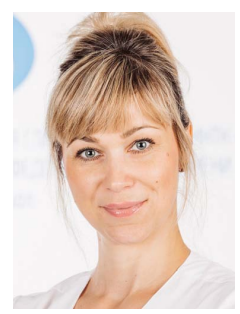

Н.В. Попова ${ }^{1}$

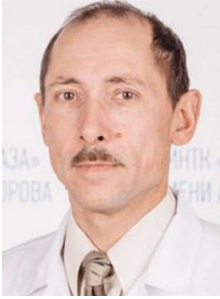

А.П. Гойдин ${ }^{1,2}$

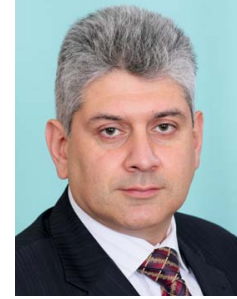

О.Л. Фабрикантов ${ }^{1,2}$

1 Тамбовский филиал ФГАУ НМИЦ «МНТН “Микрохирургия глаза” им. академина С.Н. Федорова» Министерства здравоохранения Российской Федерации

Рассказовское шоссе, 1, Тамбов, 392000, Российская Федерация

2 ФГБОУ ВПО «Тамбовский государственный университет имени Г.Р. Державина», Медицинский институт ул. Советская, 93, Тамбов, 392000, Российская Федерация

\section{PEЗЮME}

Офтальмология. 2021;18(3):399-407

Ретинопатия недоношенных $(\mathrm{PH})$ - сложная патология среди преждевременно рожденных детей. Процесс роста и формирования сосудов сетчатки достаточно сложный, и его регулируют следующие медиаторы: фактор роста фибробластов, фантор роста эндотелия сосудов (VEGF), инсулиноподобный фантор роста-1 и др. Фантор роста эндотелия сосудов наиболее тщательно изучен, его экспрессия является кислород- зависимой. Он продуцируется ретинальными перицитами, клетнами пигментного эпителия, клетками эндотелия, ретинальными глиальными клетками (клетки Мюллера). После освобождения из гипоксичных тканей VEGF связывается с эндотелиальными клетками сосудов, способствует их активации и пролиферации, что приводит к появлению ретинальных сосудов. Для решения проблемы РН необходим комплексный подход, включающий нак профилактину развития более тяжелых стадий заболевания, в результате ведущих к слабовидению и необратимой потере зрительных функций, тан и организацию диспансерного наблюдения, реабилитацию. Современная организация диспансерного наблюдения, которая основана на детальном изучении этиологии, патогенеза, а также взаимодействии лональных и системных факторов в отношении зрительных функций, позволяет значительно уменьшить инвалидизацию детей по зрению в результате данного заболевания.

Ключевые слова: ретинопатия недоношенных, сетчатка, аваскулярная зона, лазеркоагуляция

Для цитирования: Попова Н.В., Гойдин А.П., Фабрикантов О.Л. Ретинопатия недоношенных. Обзор. Офтальмология. 2021;18(3):399-407. https://doi.org/10.18008/1816-5095-2021-3-399-407

Прозрачность финансовой деятельности: Нинто из авторов не имеет финансовой заинтересованности в представленных материалах или методах

Конфликт интересов отсутствует

\section{Retinopathy of Prematurity. Review}

\author{
N.V. Popova1 ${ }^{1}$ A.P. Goydin ${ }^{1,2}$, O.L. Fabrikantov ${ }^{1,2}$
}

${ }^{1}$ Tambov branch of the S. Fyodorov Eye Microsurgery Federal State Institution

Rasskazovskoe highway, 1, Tambov, 392000, Russian Federation

${ }^{2}$ Medical Institute of the Tambov State University named after G.R. Derzhavin Sovetskaya str., 93, Tambov, 392000, Russian Federation 


\section{ABSTRACT}

Ophthalmology in Russia. 2021;18(3):399-407

Retinopathy of prematurity (RP) is a complex pathology among prematurely born children, which requires a comprehensive approach, including both prevention of the development of more severe stages of the disease, leading to poor vision and irreversible loss of visual functions, and the organization of dispensary observation and rehabilitation. Modern organization of dispensary observation, which is based on a detailed study of the etiology, pathogenesis, as well as the interaction of local and systemic factors on visual functions, can significantly reduce the visual disability of children as a result of this disease.

Keywords: retinopathy of prematurity, retina, avascular zone, laser coagulation

For citation: Popova N.V., Goydin A.P., Fabrikantov-O.L. Retinopathy of Prematurity. Review. Ophthalmology in Russia. 2021;18(3):399-407. https://doi.org/10.18008/1816-5095-2021-3-399-407

Financial Disclosure: No author has a financial or property interest in any material or method mentioned There is no conflict of interests

Ретинопатия недоношенных (РН) - вазопролиферативное заболевание, которое возникает у преждевременно рожденных детей и является в настоящее время актуальной проблемой неонатологии и офтальмологии. Роль данного заболевания в структуре формирования слабовидения и слепоты с раннего детства резко возросла за последние 10-15 лет. В нозологической структуре детской инвалидности на долю ретинопатии недоношенных приходится 20-40 \% [1].

В последнее десятилетие частота преждевременных родов составляет от 5 до $12 \%$ от числа всех родов. Внедрение и разработка в медицинскую практику новых современных технологий экстракорпорального оплодотворения, пролонгирования патологической беременности, усовершенствование первичной реанимации и интенсивной терапии недоношенных, в том числе и с экстремально низкой массой тела (ЭНМТ) при рождении (меньше 1000 г), а также ранний гестационный возраст позволяют повысить выживаемость у ранее нежизнеспособных глубоко недоношенных детей, что, в свою очередь, увеличивает вероятность появления ретинопатии [2].

Возникновение $\mathrm{PH}$ в разных регионах и странах варьирует от 17 до $35 \%$, в группе риска находится до $90 \%$ среди детей, преждевременно рожденных с ЭНМТ.. Доказано, что частота развития РН зависит не только от степени недоношенности ребенка, но также и от соматической отягощенности (мать-плод), условий содержания ребенка. Следовательно, акушеры-гинекологи, реаниматологи, неонатологи являются основным звеном в профилактике возникновения, развития и прогрессирования РН. Современные научно обоснованные протоколы выхаживания недоношенных детей позволяют свести к минимуму вероятность возникновения РН при рождении детей с массой тела выше 1500 г и сроком гестации более 32 недель. Но у детей с выраженной соматической отягощенностью и ЭНМТ, несмотря на современные условия содержания в перинатальных центрах, не исключена высокая вероятность развития $\mathrm{PH}$.

\section{ЭТИОЛОГИЯ И ПАТОГЕНЕЗ}

Этиопатогенез $\mathrm{PH}$ до настоящего времени не совсем понятен, несмотря на многочисленные исследования, указывающие на многофакторный характер этого заболевания.

Основной особенностью глаза у преждевременно рожденного ребенка является нарушение во время внутриутробного развития нормального ангиогенеза и васкулогенеза сетчатки. Уже на 14-й неделе внутриутробного развития появляются начальные признаки ретинального ангиогенеза в виде перипапиллярной сети, которая состоит из мезенхимальных веретенообразных клеток и представляет собой прообраз будущей капиллярной сети. На 14-й неделе гестации у эмбриона человека была обнаружена центральная артерия сетчатки. На втором месяце развития плода активно растет гиалоидная артерия с ответвлениями. К 16-20-й неделе внутриутробного развития начинаются процессы морфофункциональной дифференцировки в проксимальных отделах первичной капиллярной сети с формированием в первую очередь артериолярных, а затем и венулярных звеньев. Для преждевременно рожденных детей характерно наличие необлитерированных сосудов хрусталика, которые существуют до 34-й недели гестации. К 35-й неделе внутриутробного развития ретинальные сосуды достигают зубчатой линии в назальном сегменте, в темпоральном - к 40-й неделе, так как в этой части глазного дна расстояние от диска зрительного нерва (ДЗН) до зубчатой линии больше. Таким образом, чем меньше сроки гестации, тем площадь аваскулярных зон на периферии сетчатки больше $[3,4]$.

Патологическое развитие сосудов сетчатки у преждевременно рожденных детей можно условно разделить на две фазы:

1. Начальная фаза задержки физиологического развития васкуляризации.

2. Вазопролиферативная фаза (на границе аваскулярной и васкуляризированной зоны сетчатки).

Известно, что процесс роста и формирования сосудов сетчатки достаточно сложный и его регулируют следующие медиаторы: фактор роста фибробластов, фактор роста эндотелия сосудов (VEGF), инсулиноподобный фактор роста-1 и др. Фактор роста эндотелия сосудов наиболее тщательно изучен, его экспрессия является кислородзависимой. Он продуцируется ретинальными перицитами, клетками пигментного эпителия, клетками эндотелия, ретинальными глиальными клетками 
(клетки Мюллера). После освобождения из гипоксичных тканей VEGF связывается с эндотелиальными клетками сосудов, способствует их активации и пролиферации, что приводит к появлению ретинальных сосудов.

Пребывание недоношенного ребенка после рождения в условиях гипероксии приводит к снижению экспрессии ангиогенных факторов, что способствует развитию первой фазы. А нарастающая гипоксия и возрастающая метаболическая потребность развивающейся сетчатки способствуют выработке ангиогенных факторов, что приводит к возобновлению сосудистого роста. Это является началом вазопролиферативной фазы, в результате которой часто возникает неконтролируемая неоваскуляризация. Новообразованные сосуды часто являются причиной геморрагии, транссудативных выпотов, приводят к дегенеративным изменениям фоторецепторов и ганглиозных клеток сетчатки. Возможен спонтанный регресс новообразованные сосуды запустевают, в этом случае активная фаза заболевания имеет благоприятный исход. Если неоваскуляризация продолжает распространяться не только на сетчатке, но и в стекловидном теле (интравитреальная неоваскуляризация), то исход заболевания неблагоприятный и сопровождается снижением зрительных функций вплоть до слепоты [5].

Инсулиноподобный фактор роста-1 (IGF-1) является кислород-нерегулируемым, продуцируется плацентой в период внутриутробного развития. Поэтому преждевременное рождение детей приводит к резкому снижению данного фактора в сыворотке крови, что, в свою очередь, негативно отражается на развитии сосудов сетчатки, так как IGF-1 является синергистом VEGF. Низкое содержание в крови IGF-1 свидетельствует о высокой вероятности возникновения $\mathrm{PH}$ [6].

По данным многочисленных исследований высокий уровень билирубина (более 171 мкмоль/л) является одним из возможных факторов развития $\mathrm{PH}$ у преждевременно рожденных детей.

Кроме того, в ряде литературных публикаций дискутируется вопрос о связи вирусных инфекций с развитием РН. Факторами риска являются и проблемы со здоровьем матери в период беременности. Сюда следует отнести патологические изменения, которые нарушают маточно-плацентарное кровообращение и способствуют возникновению гипоксии плода (хронические заболевания женских половых органов, гестоз, кровотечение в родах). Гипоксия создает благоприятный фон для развития различных заболеваний у преждевременно рожденного ребенка, в том числе и $\mathrm{PH}$.

Обсуждается роль иммунных реакций, индуцированных S-антигеном сетчатки, как одного из патогенетических факторов развития $\mathrm{PH}$ [7].

Но, несмотря на все вышеперечисленное, большинство исследователей основными факторами, способствующими появлению и прогрессированию РН, считают гестационный возраст и массу тела при рождении $[8,9]$.

\section{КЛАССИФИКАЦИЯ}

Существует единая Международная классификация зетинопатии недоношенных (ICROP), которая состоит из 2 частей, опубликованных впервые в 1984 и 1987 гг. [10, 11]. В первой части описываются проявления активных стадий ретинопатии [10], во второй - рубцовые стадии PH [11]. Эта классификация представляет собой итог работы интернациональной группы экспертов по РН, что позволяет осмыслить всю тяжесть данного заболевания.

Согласно классификации ICOР в течении РН выделяют 3 фазы: активную, регресс и рубцовую.

Активная фаза РН подразделяется по стадиям процесса, его локализации и протяженности.

I стадия - наличие демаркационной линии на границе аваскулярной и васкуляризированной зоны сетчатки, гистологически представляющая собой скопление гиперплазированных веретенообразных клеток, имеет белесоватый цвет и располагается в плоскости сетчатки. На периферии глазного дна, перед демаркационной линией, сосуды сетчатки могут быть извиты и расширены, образовывать сосудистые ветвления, внезапно обрывающиеся, не проникающие в аваскулярную сетчатку за демаркационную линию.

II стадия - формирование на месте демаркационной линии демаркационного вала (гребня), который проминирует над плоскостью сетчатки, имеет цвет от серовато-белого до розового, что зависит от степени гиперемии за счет проникших в него сосудов. Гистологически картина представляет собой гиперплазию веретенообразных клеток с пролиферацией клеток эндотелия.

На I-II стадии заболевания возможен самопроизвольный регресс с минимальными остаточными изменениями на глазном дне в 73-80 \% случаев.

III стадия - появление экстраретинальной фиброваскулярной пролиферации в области демаркационного вала. По степени поражения и в зависимости от количества экстраретинальной фиброзной ткани с прорастанием в стекловидное тело ее подразделяют на умеренную и распространенную. В умеренную стадию резко повышается сосудистая активность в заднем полюсе глаза с образованием артериовенозных шунтов на периферии и выраженной экссудацией в стекловидное тело. Экстраретинальная пролиферация может иметь вид нежных волокон с сосудами или плотной ткани, расположенных за пределами сетчатки над валом. Если протяженность патологических изменений небольшая, то возможен самопроизвольный регресс заболевания, хотя и с более выраженными остаточными изменениями на сетчатке. Распространенность экстраретинальной пролиферации на 5 последовательных или 8 суммарных часовых меридианов характеризует развитие так называемой пороговой стадии заболевания, после этого процесс становится практически необратимым.

IV стадия - частичная отслойка сетчатки, она подразделяется на IVa стадию - без вовлечения в процесс 
макулярной зоны (так называемая экстрафовеальная частичная отслойка) и IVb стадию - с отслойкой сетчатки в макуле. Отслойка сетчатки в данных случаях носит экссудативно-тракционный характер и может развиваться как за счет серозно-геморрагического компонента, так и за счет формирующейся тракции со стороны новообразованной фиброваскулярной ткани.

V стадия - полная или тотальная отслойка сетчатки, как правило, имеющая воронкообразную форму за счет выраженной деструкции стекловидного тела, появления в нем полостей и пустот. Различают открытую, полузакрытую и закрытую форму воронкообразной отслойки сетчатки. Открытая воронка вогнутой формы простирается до диска зрительного нерва. Наиболее часто встречаемая конфигурация отслойки - закрытая, при которой передняя и задняя части воронки узкие, и отслоенная сетчатка располагается сразу за хрусталиком. Самой менее распространенной формой является полузакрытая, при которой воронка открыта в передней части и сужается в задней.

Микроскопически в отслоенной сетчатке определяются дегенерация внешних и внутренних слоев фоторецепторов и поверхностный глиоз.

IV и V стадии РН принято считать терминальными, что связано с плохим прогнозом и резким нарушением зрительных функций.

Согласно последним дополнениям, принятым в международной классификации активной $\mathrm{PH}$, имеются важные признаки, которые являются показателем активности процесса и обозначаются как «плюс-болезнь» в I-III стадии заболевания. Характерно расширение и извитость центральных и концевых сосудов сетчатки в двух и более квадрантах глазного дна и тенденция к прогрессированию заболевания.

Следует отметить, что для более полного отражения клинических проявлений РН ряд исследователей считает целесообразным введение понятия «0 стадия» - преретинопатия, для которой характерны извитые и узкие сосуды сетчатки на периферии, бледно-желтый цвет глазного дна [12].

Распространение патологического процесса на глазном дне оценивают по часовым меридианам (секторам) с 1-го по 12-й.

По локализации РН выделяют три зоны, расположенные концентрично вокруг диска зрительного нерва (ДЗН). Это обусловлено тем, что процесс васкуляризации берет начало от диска зрительного нерва по направлению к зубчатой линии. 1-я зона - окружность с центром в ДЗН и радиусом, равным удвоенному расстоянию диск-макула. 2-я зона - от края первой зоны до переднего края назальной области сетчатки (на 3-х часах в правом глазу, на 9-ти часах в левом). 3-я зона оставшаяся серповидная область сетчатки, которая располагается кпереди от второй зоны.

Использование более совершенной техники для диагностики патологии глазного дна у недоношенных детей позволило в 2005 году интернациональной группе детских офтальмологов внести дополнения в существующую классификацию [13]:

- задняя агрессивная ретинопатия недоношенных (3АРН) - наиболее опасная форма $\mathrm{PH}$, которая прогрессирует стремительно, отсутствует четкая стадийность. Минуя характерный переход от I к III стадии, быстро развивается экстраретинальная фиброваскулярная пролиферативная ткань, причем не только на границе с аваскулярной сетчаткой, но и расположенная более центрально - возле ДЗН и по ходу сосудов. В процесс вовлекается задний полюс глаза, то есть первая зона. Агрессивная РН, как правило, протекает с выраженной сосудистой активностью, резким расширением и извитостью сосудов сетчатки (во всех 4 квадрантах), образованием мощных сосудистых аркад, кровоизлияниями и экссудативными реакциями. Часто ей сопутствуют изменения в переднем отрезке глаза: ригидность зрачка, неоваскуляризация радужки. Стремительное течение ЗАРН, низкая эффективность профилактических мероприятий приводят к быстрому развитию терминальных стадий заболевания;

- выделение пре-«плюс-болезни» как промежуточной стадии извитости и расширения сосудов, предшествующей непосредственному развитию «плюс- болезни».

За активной стадией следует стадия регресса, для которой свойственно продвижение васкуляризации к периферии сетчатки, в ранее аваскулярные зоны.

Фаза регресса переходит в рубцовую фазу, которая характеризуется целым рядом изменений в заднем полюсе глаза и в периферических отделах сетчатки. Чем тяжелее протекало заболевание в активную фазу, тем выше риск возникновения грубых остаточных изменений в рубцовую фазу. Периферические изменения включают в себя патологическую пигментацию, истончение, решетчатую дегенерацию сетчатки, разрывы и отслойку сетчатки, формирование телеангиоэктазий и аркад сосудов, изменения в заднем полюсе: деформация ДЗН, образование складок сетчатки и субретинальных мембран, дислокация макулы, тракционно-регматогенная отслойка сетчатки. Может быть вовлечен в процесс и передний отрезок глаза, что проявляется помутнением роговицы, формированием передних и задних синехий, мелкой передней камерой, осложненной катарактой, вторичной глаукомой [14].

\section{КЛИНИЧЕСКАЯ КАРТИНА}

Жалобы пациент и родители не предъявляют. При физикальном обследовании симптомы начальных стадий ретинопатии недоношенных выявить невозможно. Заболевание выявляется при активном скрининге преждевременно рожденных детей группы риска методом непрямой бинокулярной офтальмоскопии или при обследовании с помощью ретинальной камеры. Оценить выявленные изменения на глазном дне возможно в соответствии с классификацией активной $\mathrm{PH}$. 


\section{ДИАГНОСТИКА И СКРИНИНГ РН}

Для своевременного выявления РН необходима организация офтальмологических осмотров и скрининга преждевременно рожденных детей, находящихся в группе риска. В нашей стране осмотр офтальмолога должны пройти все недоношенные дети, рожденные ранее 35-й недели гестационного возраста (ГВ) и/или с массой тела менее 2000 г, в некоторых неонатальных центрах и странах включены в группу риска дети с гестационным возрастом при рождении ранее 32 -й недели и массой тела до 1500 г [15].

Начало скрининга активной РН зависит от постконцептуального возраста ребенка (ПВК). Постконцептуальный возраст - полный возраст ребенка в неделях с начала последнего менструального цикла матери, определяющий степень его зрелости.

ПВК = гестационный возраст при рождении + хронологический возраст жизни (недели).

Дети, рожденные в ГВ 22-26 недель, должны быть осмотрены офтальмологом на 31-й неделе ПКВ, в ГВ 27-31 неделя - с 4-й недели жизни, в ГВ 32 недели и более - с 3-й недели жизни.

Наиболее распространенным методом диагностики активной РН является обратная бинокулярная офтальмоскопия в условиях медикаментозного мидриаза с использованием линзы +20 дптр. Для лучшей визуализации периферических отделов глазного дна целесообразно применять стерильные инструменты - векорасширитель и склеральный депрессор, которые предназначены для применения в неонатальной практике.

Как правило, уже одного осмотра достаточно для постановки диагноза активной РН. При выявлении риска развития РН - наличие аваскулярных зон (незаконченная васкуляризация сетчатки) без признаков РН и «плюс-болезни» - следующий осмотр офтальмологом должен быть проведен через 2 недели с момента первичного осмотра. Далее осмотры с интервалом в 2 недели осуществляют до полной васкуляризации сетчатки или до развития $\mathrm{PH}$.

Если были выявлены клинические признаки активной $\mathrm{PH}$, то осмотры осуществляются каждую неделю до самостоятельной остановки заболевания или до определения показаний к лазеркоагуляции сетчатки. При ЗАРН с локализацией процесса в первой зоне глазного дна офтальмологические осмотры проводят каждые 3 дня.

Завершают скрининговые осмотры по достижении ПКВ ребенком в возрасте 42-44 недели, а также по окончании васкуляризации сетчатки на периферии, то есть при полном регрессе активной $\mathrm{PH}$.

С появлением цифровой ретиноскопии с использованием ретинальной педиатрической камеры RetCam Shuttle стало возможным не только объективно оценивать изменения на глазном дне, но и сохранять результаты в банке данных, проводить дистанционное консультирование. Исследование недоношенного ребенка с помощью ретинальной камеры дает возможность оценивать передний отрезок глазного яблока, а именно состояние угла передней камеры, радужки, хрусталика, а также в заднем отрезке выявить патологию стекловидного тела, уточнить высоту и локализацию отслоенной сетчатки, наличие витреальных тяжей и их связь с другими структурами [16]. С помощью современных ретинальных камер появилась возможность проводить флюоресцентную ангиографию (ФАГ) у недоношенных детей при РН, что позволяет получать сведения о том, как протекает заболевание, о локализации патологического процесса, оценивать результаты лечения. Некоторые исследователи считают данный метод совершенно безопасным при правильно подобранной дозе флюоресцеина, другие же предусматривают возможные осложнения, так как исследование является инвазивным, и проводить его стоит только по определенным показаниям и в спорных сложных диагностических ситуациях.

Большое значение в последнее время приобрели методы исследования, с помощью которых стало возможно более детально визуализировать макулярную зону у недоношенных детей, так как ее состояние в целом определяет зрительные функции. Основным таким методом является оптическая когерентная томография (ОКТ), достоинством которой является неинвазивность, высокая разрешающая способность, возможность получать томограммы в конкретно заданном участке сетчатки, а также количественная и качественная оценка витреоретинального интерфейса [17].

По данным многочисленных исследований доказано, что толщина перипапиллярной сетчатки взаимосвязана с массой тела ребенка при рождении. Центральная толщина сетчатки в макулярной области у недоношенных детей по сравнению с доношенными больше. Доказана зависимость между толщиной сетчатки, остротой зрения и рефракцией. Ряд авторов считает, что аномалии в фовеолярной области являются маркерами недоношенности [18].

Среди функциональных методов оценки состояния зрительного анализатора у детей раннего возраста особое место занимают электрофизиологические исследования - ЭРГ и ЗВП.

ЭРГ используется для количественной оценки функционального состояния нейронов сетчатки, причем каждый из компонентов ЭРГ генерируется разными структурами сетчатки. Негативная волна «а» отражает функцию фоторецепторов как начальную часть позднего рецепторного потенциала, позитивная волна «б» - электрическую активность биполяров и клеток Мюллера. При определенных условиях регистрации (при больших длительностях стимулирующего света) можно зафиксировать и волну «с» - показатель состояния транспигментного потенциала эпителия. Следует учесть то, что в современной литературе практически нет данных о показателях ЭРГ в активный период $\mathrm{PH}$, а имеющиеся сведения достаточно противоречивы. На небольшом клиническом материале показано, что слой фоторецепторов при РН является основной зоной 
дисфункции сетчатки. В стадии регресса РН конфигурация волн в фотопических и скотопических состояниях изменяется в сторону роста амплитуды волн «а» и «б» [19].

Доказано, что РН часто сопутствует поражение центральной нервной системы, поэтому регистрация ЗВП имеет в данных случаях большое значение, так как отражает функциональное состояние всего зрительного пути и зрительных корковых центров.

ЗВП регистрируется на паттерн-стимул или вспышку в виде последовательных компонентов, ведущим из которых является Р100, уже в первые дни жизни ребенка. На 22-23-й неделе гестационного возраста ЗВП имеет вид негативной волны с латентностью около 300 мс. С 30-34-й недели ГВ перед ней появляется позитивный компонент с латентностью 200 мс. Через некоторое время после рождения недоношенного ребенка данные ЗВП оказываются более зрелыми, чем у доношенных сверстников, что доказывает зависимость развития зрительного анализатора от внешних воздействий.

\section{ПРОФИЛАКТИКА И ЛЕЧЕНИЕ РАННИХ СТАДИЙ РН}

Предотвращение невынашивания беременности и преждевременных родов, совершенствование неонатальных протоколов выхаживания недоношенных детей, особенно с экстремально низкой массой тела, является основной задачей акушеров-гинекологов, реаниматологов и неонатологов и представляет собой профилактику $\mathrm{PH}$.

Период ранней постнатальной адаптации у недоношенных детей протекает достаточно тяжело, что связано с морфофункциональной незрелостью организма и требует проведения интенсивных лечебных мероприятий. Не только соматическая патология, но и интенсивная терапия недоношенного ребенка, которая проводится по жизненным показаниям, может негативно влиять на развитие и прогрессирование $\mathrm{PH}$ [20].

Консервативное или медикаментозное лечение, применяемое для профилактики развития $\mathrm{PH}$, имеет крайне низкую эффективность, что доказано на немногочисленной группе преждевременно рожденных детей.

В настоящее время единственным доказанным и признанным методом профилактики прогрессирования $\mathrm{PH}$ и перехода ее в более тяжелые стадии, а также способом сохранения зрительных функций является лазеркоагуляция (ЛК) сетчатки. Имеются многочисленные данные отечественной и зарубежной литературы, которые подробно излагают показания к проведению лазеркоагуляции, сроки, методики выполнения, недостатки и преимущества различных вариантов лазеркоагуляции [21, 22].

Показания к выполнению лазеркоагуляции:

- любая стадия РН с локализацией в 1-й зоне глазного дна с «плюс-болезнью»;

- III стадия РН с локализацией в 1-й зоне без «плюсболезни»;

- III стадия РН с распространением на 5 последовательных часовых поясов с «плюс-болезнью» при локализации во II-III зоне.
В зависимости от формы течения РН и сроков проведения ЛК у недоношенного ребенка эффективность лечения варьирует от 60 до 98 \%. Индуцированный регресс заболевания с минимальными остаточными изменениями на сетчатке и сохранением высоких зрительных функций возможен практически в 100 \% при своевременно проведенной и методически правильной лазеркоагуляции при РН с локализацией процесса во II-III зоне глазного дна. При ЗАРН и РН I зоны эффективность лазерного лечения существенно ниже, что объясняется другими механизмами патогенеза заболевания.

Абсолютных противопоказаний к проведению ЛК у детей с РН не существует. К относительным противопоказаниям относят: дакриоцистит новорожденных, тяжелое соматическое состояние ребенка, сопряженное с высоким риском проведения лазерного лечения под общей анестезией.

В основе механизма действия ЛК лежит деструкция ишемических зон аваскулярной сетчатки. В результате коагуляции происходит перерождение сетчатки в тонкую фиброзную ткань с атрофией пигментного эпителия, обнажением мембраны Бруха и атрофией подлежащих сосудов хориоидеи, капилляров.

Методика лазеркоагуляции заключается в нанесении коагулятов на аваскулярную зону сетчатки. В ряде случаев при наличии «плюс-болезни» в 1-3-м сегментах возможно выполнение секторальной коагуляции или нанесение коагулятов не по всей площади аваскулярной зоны, а всего лишь в несколько рядов вдоль демаркационного вала. При ЗАРН лазером должна быть обработана не только аваскулярная сетчатка, но и зоны сетчатки, которые находятся центральнее вала. Причем тяжелые формы РН требуют применения сливной коагуляции, которая позволяет более плотно заблокировать ишемические зоны сетчатки [21]. Но слишком массивная ЛК с образованными в дальнейшем массивными сливными зонами атрофии может являться причиной развития нежелательных осложнений, таких как глаукома, повреждения переднего отрезка глаза, что важно учитывать при проведении лечения.

«Золотой стандарт» лечения активной $\mathrm{PH}$ - транспупиллярная лазеркоагуляция сетчатки [21], которая проводится с использованием лазерной офтальмологической системы PASCAL Photocoagulator (Ohtimedica, США) с применением контактной офтальмологической линзы Quad Pediatric Fundus Lens (Volk). Предоперационная подготовка пациента включает осмотр педиатра и анестезиолога, инстилляцию средства для расширения зрачка. Коагуляция проводится под эндотрахеальным наркозом в положении ребенка на боку на специальном столикеподставке с регулируемой высотой подъема.

Алгоритм выполнения ЛК состоит из нескольких этапов: сначала необходимо провести тестирование коагулята в режиме одиночного импульса, а затем уже на основании тестирования определить энергетические параметры паттернов и обработать всю площадь 
аваскулярной сетчатки. Общее количество коагулятов зависит от площади ишемических зон [22].

Преимущества паттерн-ЛК:

1) значительное сокращение времени процедуры, которое достигается за счет нанесения группы коагулятов;

2) исключение возможности перекрывания лазерных коагулятов между собой, отсутствие эффекта гиперкоагуляции;

3) обеспечение высокой дозированности лазерного воздействия (оптимальное соотношение мощности и экспозиции со снижением суммарной плотности энергии лазерного излучения);

4) возможность выполнения ЛК вне зависимости от площади и локализации патологического процесса аваскулярной сетчатки, за один сеанс.

Оценка эффективности проведенной ЛК проводится через 7-10 дней после лечения. Показатели эффективности, которые указывают на регресс заболевания:

- клинические признаки: уменьшение, уплощение и исчезновение демаркационного вала, регресс экстраретинальной пролиферации, уменьшение и исчезновение отека сетчатки;

- морфометрические признаки: уменьшение диаметра и извитости магистральных сосудов заднего полюса глаза.

Об отсутствии эффекта от проведенного лечения свидетельствует формирование грубых деформаций в заднем полюсе глаза, серповидных складок, отслойка сетчатки.

\section{ТЕРАПИЯ С ПРИМЕНЕНИЕМ ФАКТОРОВ РОСТА СОСУДОВ АНТИ-VEGF}

Деструкция клеточных элементов аваскулярной сетчатки, которые индуцируют VEGF и способствуют формированию экстраретинальной фиброваскулярной пролиферации, является патогенетически оправданной при РН и в какой-то мере достигается лазеркоагуляцией. Для того чтобы лазеркоагуляция имела хороший эффект, проводить ее необходимо в ранние сроки. Но существуют формы РН, такие как ЗАРН, РН 1-й зоны, когда лазеркоагуляция малоэффективна. Если VEGF продуцируется только в сетчатке, то лазерная деструкция клеточных элементов может привести к остановке процесса, но если VEGF присутствует и в стекловидном теле, коагуляция неэффективна.

В таких случаях необходимо применять новые патогенетически обоснованные методы лечения. Одним из таких методов является применение ингибиторов факторов роста сосудов, которые могут быть использованы во 2-й фазе патогенеза $\mathrm{PH}-$ фазе пролиферации. С этой целью используются ингибиторы эндотелиального сосудистого фактора роста: ранибизумаб (Луцентис) - фрагмент человеческого моноклонального антитела к фактору роста эндотелия сосудов А; бевацизумаб (Авастин) - гуманизированное моноклональное антитело к фактору роста эндотелия сосудов. Дозы препарата подбираются индивидуально.
Антиангиогенная терапия может быть альтернативой лазерному лечению РН 1-й зоны у детей с ЭНМТ, что доказано в многоцентровых рандомизированных исследованиях, которые были проведены в США. Преимуществом данного вида лечения является возможность последующей васкуляризации аваскулярных зон, чего не происходит после лазеркоагуляции в связи с полным рубцовым замещением аваскулярной сетчатки.

Существует мнение о возможном использовании анти-VEGF терапии только после лазеркоагуляции сетчатки при выраженной сосудистой активности и продолжающейся экстраретинальной пролиферации [23], но имеются работы, в которых рассматривается возможность введения препаратов без предварительной коагуляции сетчатки при ЗАРН и РН 1-й зоны с «плюсболезнью» [24].

Преимуществами данного вида лечения являются: купирование сосудистой активности, регресс новообразованных сосудов, инволюция персистирующих фетальных сосудов, уменьшение экссудации, рассасывание витреальных и ретинальных кровоизлияний, а также возможность продолженной ретинальной васкуляризации в периферических отделах сетчатки. К сожалению, не исключена вероятность поздней активации процесса, что требует дальнейшего исследования причин, а также более тщательного контроля за недоношенными детьми после проведенного лечения. Тревожность неонатологов вызывает и возможное развитие побочного негативного действия препарата, который попадает в системный кровоток, на развитие головного мозга, почек и других внутренних органов [25].

При низкой эффективности или отсутствии эффекта от лазеркоагуляции сетчатки у недоношенных детей развиваются тяжелые рубцовые формы РН, что приводит к необратимой потере зрительных функций. При развитии отслойки сетчатки и тяжелых рубцовых стадиях РН рекомендуется проводить хирургическое вмешательство. В последние годы при терминальных стадиях РН выполняется закрытая двух- или трехпортовая витрэктомия с использованием современного микрохирургического инструментария: 23G (0,6 мм), 25G (0,5 мм), 27G (0,4 мм).

$\mathrm{y}$ пациентов с частичной или тотальной отслойкой сетчатки по типу открытой воронки хирургическое вмешательство проводят с оптико-реконструктивной целью, когда имеется возможность улучшения зрительных функций. При отслойке сетчатки по типу закрытой воронки или полузакрытой с выраженным фиброзом и трансретинальными сосудистыми мембранами выполняют ленсвитрэктомию с органосохранной целью, что носит реконструктивный характер [26].

Показания к хирургическому лечению и сроки выполнения при тяжелых стадиях $\mathrm{PH}$ широко варьируют. В активной фазе РН при появлении начальных признаков отслойки сетчатки, несмотря на проведенную лазеркоагуляцию сетчатки, целесообразно решать вопрос 
о проведении микрохирургического вмешательства, сохраняя прозрачный хрусталик. Показания к оперативному лечению в рубцовую фазу РН: V стадия на обоих глазах при сохраненном светоощущении; IV стадия $\mathrm{PH}$ при выраженных фиброзных изменениях стекловидного тела с наличием витреоретинальной тракции или локализация плотных мембран в оптической зоне; синдром мелкой передней камеры с развитием иридо- и/или ленсроговичного контакта [26].

Относительные противопоказания к оперативному лечению: отсутствие светоощущения и нерегистрируемые зрительно-вызванные корковые потенциалы (ЗВП), протяженный ретинодиализ, монокулярное поражение (IV-V стадия РН на одном глазу без изменений переднего отрезка глаза, ухудшающих качество жизни пациента). Абсолютные противопоказания к операции в активной и рубцовой фазе $\mathrm{PH}$ : тяжелое соматическое состояние ребенка, невозможность проведения наркоза [26].

Анатомический и функциональный результат может снижаться в отдаленном периоде за счет репролиферации и появления вторичных разрывов сетчатки. Решение вопроса о повторных хирургических вмешательствах остается открытым до настоящего времени.

\section{СОПУТСТВУЮЩАЯ ПАТОЛОГИЯ У ДЕТЕЙ С РН, РЕФРАКЦИОННЫЕ И ГЛАЗОДВИГАТЕЛЬНЫЕ НАРУШЕНИЯ}

Ведущую роль в нарушении зрения у недоношенных детей играет сопутствующая патология ЦНС: гидроцефалия, гипоксия и ишемия мозга и др. Последствия данных состояний могут влиять на развитие зрения, а своевременная диагностика уровня поражения позволяет прогнозировать зрительные функции и определяет тактику медицинской и социальной реабилитации.

Одним из факторов, который оказывает влияние на остроту зрения у детей с $\mathrm{PH}$, является рефракционный. Для 50 \% недоношенных детей характерна миопическая рефракция, в отличие от доношенных детей, для которых на первом году жизни характерна гиперметропическая рефракция в 2,0-4,0 дптр. Развитию миопии способствует преждевременное рождение, которое ассоциируется с увеличенной кривизной роговицы, повышенной преломляющей силой хрусталика и уменьшением глубины передней камеры. Миопия у детей с $\mathrm{PH}$ носит стационарный характер в отличие от миопии у недоношенных детей без $\mathrm{PH}$, которая исчезает к возрасту 1 год. Взаимосвязь степени недоношенности и миопии не определяется, но, по данным некоторых работ, у детей с массой тела при рождении менее 750 г миопическая рефракция встречается в 3,2 раза чаще, чем у детей с массой тела 751-1000 г [27].
Дискутируется вопрос о роли лазеркоагуляции, которая проводится в активную стадию РН, в формировании миопии. По некоторым данным, дети, в анамнезе которых было лазерное лечение, в 3 раза чаще имели миопическую рефракцию по сравнению с детьми при самопроизвольном регрессе. Однако механизм воздействия профилактического лечения на формирование рефракции при $\mathrm{PH}$ окончательно не изучен. Известно, что миопия негативно влияет на структурное и функциональное развитие макулярной зоны, что сказывается на остроте зрения [27].

Дети с РН довольно часто имеют глазодвигательные нарушения, такие как нистагм, косоглазие, что связано с высоким процентом перинатального поражения ЦНС. Основные факторы развития косоглазия - недоношенность, экстремально низкая масса тела при рождении, тяжесть РН. Значительную роль в возникновении косоглазия играет неврологическая патология. У детей с $\mathrm{PH}$ косоглазие развивается рано - на первом году жизни. Формы косоглазия многообразны: первичное содружественное, рефракционное, анизометропическое, паралитическое, вторичное (псевдокосоглазие). Чаще всего при РН встречается вторичное и псевдокосоглазие $(42,6 \%)$, которое возникает в результате имеющихся тракционных изменений сетчатки и эктопии макулы [28]. При асимметричном поражении в 29,6 \% возникает анизометропическое косоглазие. Возникновение паралитического косоглазия при РН обусловлено поражением ядер или стволов глазодвигательного, блокового и отводящего нерва, а также поражением этих нервов в глазных мышцах или самих мышц. Многообразие форм косоглазия у детей с $\mathrm{PH}$ требует разработки определенного подхода в наблюдении и лечении.

Следующим глазодвигательным нарушением у детей с РН является нистагм, который может быть глазного и/или центрального происхождения. При PH III-IV степени и при низкой остроте зрения нистагм, как правило, глазной; центральная природа нистагма, обусловленная нарушением регуляции глазодвигательного аппарата, сопровождается высокой остротой зрения и минимальными изменениями на глазном дне [29].

Ретинопатия недоношенных в настоящее время остается сложной проблемой, для решения которой необходим комплексный подход, который включает в себя и профилактику развития тяжелых форм $\mathrm{PH}$, приводящих к необратимому снижению зрительных функций, и организацию диспансерного наблюдения с дальнейшей реабилитацией.

\section{УЧАСТИЕ АВТОРОВ:}

Попова Н.В., Фабрикантов О.Л. - концепция и дизайн исследования, научное редактирование;

Попова Н.В., Гойдин А.П. - написание текста, оформление библиографии, подготовка иллюстраций.

\section{ЛИTЕРATYPA/REFERENCES}

1. Сидоренко Е.И., Асташева И.Б. Ретинопатия недоношенных как проблема современной офтальмологии. Российская педиатрическая офтальмология. 2007;4:4-5. [Sidorenko E.I., Astasheva I.B. Retinopathy of prematurity is the problem of modern ophthalmology. Russian pediat- ric ophthalmology = Rossiiskaya pediatricheskaya oftal'mologiya. 2007;4:4-5 (In Russ.)]

2. Катаргина Л.А., Коголева Л.В. Ретинопатия недоношенных. Под ред. В.В. Нероева. М.: ГЭОТАР-Медиа; 2009:27-61. [Katargina L.A., Kogoleva L.V. Reti- 
nopathy of prematurity. Ed. by V.V. Neroev. Moscow: GEOTAR-Media; 2009:27-61 (In Russ.)]

3. Федоров А.А. Пренатальное развитие сосудов сетчатой оболочки глаза. Вестник офтальмологии. 2003;4:59-63. [Fedorov A.A. Prenatal development of human retinal vessels. Annals of ophthalmology = Vestnik oftal'mologii. 2003;4:59 63 (In Russ.)]

4. Yang C.M., Liu K.R., Chen M.S. Ocular fundus examination in premature infants. J Formos Med Assoc. 1991;90(5):480-486.

5. Yokoi T., Yokoi T., Kobayashi Y. Risk factors for recurrent fibrovascular proliferation in aggressive posterior retinopathy of prematurity after early vitreous surgery. Am J. Ophthalmol. 2010;150(1):10-15. DOI: 10.1016/j.ajo.2010.02.005

6. Hellström A., Engström E., Hård AL, Postnatal serum insulin-like growth factor I deficiency is associated with retinopathy of prematurity and other complications of premature birth. Pediatrics. 2003;112(5):1016-1020.

7. Катаргина Л.А., Слепова А.С., Скрипец П.П. Гуморальный иммунный ответ на S-антиген сетчатки у недоношенных детей и его роль в развитии и течении ретинопатии недоношенных. Вестник офтальмологии. 2003;119(1):20-22. [Katargina L.A., Slepova A.S., Skripets P.P. Humoral immune response to the retinal s-antigen in premature babies and its role in the development and course of retinopathy in such babies. Annals of ophthalmology = Vestnik oftal'mologii. 2003;119(1):20-22 (In Russ.)].

8. Holmstron G., Broberger U., Thomassen P. Neonatal risk factor in retinopathy of prematurity- a population-based study. Acta Ophthalmol Scand. 1998;76:204-207.

9. Seiberth V., Lindercamp O. Risk factor in retinopathy of prematurity. Br. J Ophthalmol. 2000;214:131-135.

10. International Committee for the Classification of the Late Stages of ROP. An International Classification of Retinopathy of Prematurity. Arch. Ophthalmol. 1984;102:1130-1134.

11. International Committee for the Classification of the Late Stages of ROP. An International Classification of Retinopathy of Prematurity II. The Classification of retinal detachment. Arch. Ophthalmol. 1987;105:906-912.

12. Терещенко А.В., Белый Ю.А., Трифаненкова И.Г. Рабочая классификация ранних стадий ретинопатии недоношенных. Офтальмохирургия. 2008;1:3234. [Tereshchenko A.V., Belyi Yu.A., Trifanenkova I.G. Classification of the early stages of retinopathy of prematurity. Ophthalmosurgery $=$ Oftal'mokhirurgiya. 2008;1:32-34 (In Russ.)].

13. The International Classification of Retinopathy of Prematurity Revisited. Arch. Ophthalmol. 2005;123(7):991-999. DOI: 10.1001/archopht.123.7.991

14. Hitter H.M., Rhodes L.M., McPerson. Anterior segment abnormalities in cicatricial retinopathy of prematurity. Ophthalmology. 1979;86:803-816.

15. Федеральные клинические рекомендации «Диагностика, мониторинг и лечение активной фазы ретинопатии недоношенных» (Национальный протокол). Российская педиатрическая офтальмология. 2015;1:54-60. [Federal clinical recommendations "Diagnosis, observation and treatment of active stage of retinopathy of prematurity" (National report). Russian pediatric ophthalmology = Rossiiskaya pediatricheskaya oftal'mologiya. 2015;1:54-60 (In Russ.)].

16. Chiang M.F., Keenan J.D., Starren J. Accuracy and reliability of remote retinopathy of prematurity diagnosis. Arch. Ophthalmol. 2006;124:322-327. DOI: 10.1001/archopht.124.3.322

17. Мосин И.М. Оптическая когерентная томография. В кн. Клиническая физиология зрения. Под ред. А.М. Шамшиновой. М.: МБН: 2006;785-858.

\section{СВЕДЕНИЯ ОБ АВТОРАХ}

Тамбовский филиал ФГАУ НМИЦ «МНТК “Микрохирургия глаза” им. академика С.Н. Федорова» Министерства здравоохранения Российской Федерации Попова Наталия Викторовна

врач-офтальмолог

Рассказовское шоссе, 1, Тамбов, 392000, Российская Федерация

Тамбовский филиал ФГАУ НМИЦ «МНТК “Микрохирургия глаза” им. академика С.Н. Федорова» Министерства здравоохранения Российской Федерации кафедра офтальмологии Медицинского института ФГБОУ ВПО «Тамбовский государственный университет имени Г.Р. Державина»

Гойдин Андрей Павлович

заместитель директора по клинико-экспертной работе; старший преподаватель Рассказовское шоссе, 1, Тамбов, 392000, Российская Федерация

ул. Советская, 93, Тамбов, 392000, Российская Федерация

Тамбовский филиал ФГАУ НМИЦ «МНТК “Микрохирургия глаза” им. академика С.Н. Федорова» Министерства здравоохранения Российской Федерации кафедра офтальмологии Медицинского института ФГБОУ ВПО «Тамбовский государственный университет имени Г.Р. Державина»

Фабрикантов Олег Львович

доктор медицинских наук, директор; заведующий кафедрой

Рассказовское шоссе, 1, Тамбов, 392000, Российская Федерация

ул. Советская, 93, Тамбов, 392000, Российская Федерация
[Mosin I.M. Optical coherence tomography. In: Clinical visual visiology. Ed. by A.M. Shamshinova. Moscow: MBN; 2006:785-858 (In Russ.)].

18. Hammer D.X., Iftimia N.V., Ferguson R.D. Foveal Fine Structure in retinopathy of prematurity: an adaptive optics Fourier domain optical coherence tomography study. Invest Ophthalmol. Vis. Sci. 2008;49(5):2061-2070. DOI: 10.1167/iovs.07-1228

19. Mets M.B., Smith V.C., Pocorny J. Postnatal retinal development as measured by the electroretinogram in preterm infants. Documenta Ophthalmologica. 1995;90:111-127.

20. Асташева И.Б., Кан И.Г., Дегтярев Д.Н. Особенности развития и течения ретинопатии недоношенных при использовании новых подходов к респираторной терапии на этапе первичной реанимации новорожденных. Вестник Оренбургского государственного университета 2014;173(12):17-19. [Astasheva I.B., Kan I.G., Degtyarev D.N. Features of development and course of retinopathy of prematurity with the use of new approaches to respiratory therapy on stage o neonatal resuscitation. Annals of Orenburg State University = Vestnik Orenburgskogo gosudarstvennogo universiteta. 2014;173(12):17-19 (In Russ.)].

21. Асташева И.Б., Сидоренко Е.И., Аксенова И.И. Лазеркоагуляция в лечении различных форм ретинопатии недоношенных. Вестник офтальмологии. 2005;121(2):31-34. [Astasheva I.B., Sidorenko E.I., Aksenova I.I. Laser coagulation in the treatment of different type retinopathy of prematures. Annals of ophthalmology = Vestnik oftal'mologii. 2005;121(2):31-34 (In Russ.)].

22. Lira R.P., Calheiros A.B., Barbosa M.M. Efficacy and safety of green laser photocoagulation for threshold retinopathy of prematurity. Arg. Bras. Ophthalmol. 2008;71(1):49-51.

23. Chung E.J., Kim J.H., Ahn H.S. Combination of laser photocoagulation and intravitreal bevacizumab (Avastin) for aggressive zone I retinopathy of prematurity. Graefes Arch Clin Exp Ophthalmol. 2007;245(11):1727-1730. DOI: 10.1007/s00417007-0661-y

24. Dorta P., Kychenthal A. Treatment of type 1 retinopathy of prematurity with intravitreal bevacizumab (Avastin). Retina. 2010;30(4 Suppl):24-31. DOI: 10.1097/ IAE.0b013e3181ca1457

25. Gaynon M.W. Rethinking STOP-ROP: is it worthwhile trying to modulate excessive VEGF levels in prethreshold ROP eyes by systemic intervention? A review of the role of oxygen, light adaptation state, and anemia in prethreshold ROP. Retina. 2006;26(7 Suppl.):18-23. DOI: 10.1097/01.iae.0000244292.86627.1e

26. Баранов А.В. Хирургическое лечение поздних стадий ретинопатии недоношенных - последний шанс видеть. Сообщение 2. Анализ функциональных результатов. Вестник офтальмологии. 2012;128(4):19-25. [Baranov A.V. Surgical treatment of advanced retinopathy of prematurity - last chance for vision. Report 2. Analysis of functional results. Annals of ophthalmology $=$ Vestnik oftal'mologii. 2012;128(4):19-26 (In Russ.)].

27. Teed R.G., Saunders R.A. Retinopathy of prematurity in extremely premature infants. J.AAPOS. 2009;13(4):370-373.

28. Ковалевская И.С. Особенности диагностики и лечения косоглазия у детей с ретинопатией недоношенных. Российская педиатрическая офтальмология 2014;4:24. [Kovalevskaya I.S. Characteristics of diagnosis and treatment of strabismus in children with retinopathy of prematurity. Russian pediatric ophthalmo$\operatorname{logy}=$ Rossiiskaya pediatricheskaya oftal'mologiya 2014;4:24 (In Russ.)].

29. Пальчик А.Б., Федорова Л.А., Понятишин А.Е. Неврология недоношенных детей. М.: МЕДпресс-информ, 2010;31-45. [Pal'chik A.B., Fedorova L.A., Ponyatishin A.E. Neurology of premature born children. Moscow: MEDpress-inform, 2010; 31-45 (In Russ.)].

\section{ABOUT THE AUTHORS}

Tambov branch of the S. Fyodorov Eye Microsurgery Federal State Institution Popova Nataliya V.

ophthalmologist

Rasskazovskoe highway, 1, Tambov, 392000, Russian Federation

Tambov branch of the S. Fyodorov Eye Microsurgery Federal State Institution Medical Institute of the Tambov State University named after G.R. Derzhavin Goydin Andrey P.

deputy director; senior lecturer

Rasskazovskoe highway, 1, Tambov, 392000, Russian Federation

Sovetskaya str., 93, Tambov, 392000, Russian Federation

Tambov branch of the S. Fyodorov Eye Microsurgery Federal State Institution Medical Institute of the Tambov State University named after G.R. Derzhavin Fabrikantov Oleg L.

$\mathrm{MD}$, director, head of ophthalmological department

Rasskazovskoe highway, 1, Tambov, 392000, Russian Federation

Sovetskaya str., 93, Tambov, 392000, Russian Federation 